\title{
Techno-economic analysis of hydrogen production from PV plants
}

\author{
Angelica Liponi $^{1}$, Andrea Baccioli $^{1}$, Lorenzo Ferrari ${ }^{1,{ }^{*}}$, and Umberto Desideri ${ }^{1}$ \\ ${ }^{1}$ Department of Energy, Systems, Territory and Construction Engineering, University of Pisa, Pisa, Italy
}

\begin{abstract}
Hydrogen production through electrolysis from renewable sources is expected to play an important role to achieve the reduction targets of carbon dioxide emissions set for the next decades. Electrolysers can use the renewable energy surplus to produce green hydrogen and contribute to making the electrical grid more stable. Hydrogen can be used as medium-long term energy storage, converted into other fuels, or used as feedstock in industry thus contributing to decarbonise hard-to-abate-sectors. However, due to the intermittent and variable nature of solar and wind power, the direct coupling of electrolysers with renewables may lead to high production fluctuations and frequent shutdowns. As a consequence, accelerated electrolyser degradation and safety issues related to low load operation may arise. In this study, simulations of hydrogen production with an electrolyser fed by a PV system are performed in Matlab for a reference year. The effect of PV power fluctuations on the electrolyser operation and production is investigated. The impact of the electrolyser size for a fixed nominal power of the PV plant is also analysed from both energetic and economic points of view.
\end{abstract}

\section{Introduction}

In order to keep global warming below $2^{\circ} \mathrm{C}$ and limit climate changes, carbon emissions must be drastically reduced. As a consequence, the share of renewable energies (RE) in electricity production is increasing with the associated need to cope with their variability and unpredictability. In this context, hydrogen production through electrolysis from RE can play a key role. Electrolysers can use the RE overproduction and contribute to making the electrical grid more stable. The green hydrogen produced can be used as energy storage, converted into other compounds (e.g. power to fuel [1]) such as methane, methanol, or ammonia or used as feedstock in the industry contributing to the decarbonize this sector.

Water electrolysis technologies can be divided into low-temperature (alkaline and polymer electrolyte membrane (PEM) electrolysers) and high-temperature electrolysers (solid oxide electrolysers). Alkaline electrolysers are the most mature and widespread technology [2]. However, they have some limitations that must be taken into account when operating with fluctuating REs. They must operate in the range of 20$100 \%$ of the nominal power. Indeed, there is the risk of formation of potentially flammable mixtures of hydrogen and oxygen due to the diffusion of these gases through the membranes when the operating current is relatively low [3]. Furthermore, the number of start-ups and stops must be limited since it is associated with a reduction of the electrolyser lifetime [3].

Solar photovoltaics (PV), along with wind energy, are the most widespread REs and are expected to increase in the next years. However, their intermittent and unpredictable nature can affect the electrolyser operation and lifetime.

In recent years, several studies dealt with the coupling of PV power and hydrogen production through electrolysis. Gutiérrez-Martín et al. [4] proposed two configurations for an off-grid PV-electrolyser system: direct coupling and battery-assisted electrolysis and found hydrogen production costs of around 6-7 $€ / \mathrm{kg} \mathrm{H}_{2}$. They asserted that batteries can support cost reductions by increasing the capacity factors of electrolysers, depending on the specific costs of these components. Schnuelle et al. [5] developed a model for both alkaline and PEM electrolysers and performed dynamic simulations with PV and wind power input datasets. They found that the better operational flexibility of PEM is generally not advantageous in terms of hydrogen production quantities and hydrogen production costs. Yates et al. [6] made a techno-economic analysis of stand-alone hydrogen production system from PV energy using Monte-Carlobased uncertainty analysis; they found that the most important drivers of the $\mathrm{LCOH}$ are system size, capital cost, and electrolyser efficiency. Khalilnejad et al. [7] performed an optimization of a directly coupled PVelectrolyser system with a hydrogen storage tank to maximum hydrogen production of electrolyser with minimum excess power production of a PV system and minimum power transfer loss finding the optimal PV size.

The choice of the relative sizes of the electrolyser and the PV plant is a key issue, made complicated by the high variability of solar energy. In this study, a system consisting of an alkaline electrolyser fed by a PV plant for hydrogen production is investigated. Annual simulations

\footnotetext{
Corresponding author: lorenzo.ferrari@unipi.it
} 
for different electrolyser sizes are performed for a case study to analyse the size effect on some key performance indicators such as the total hydrogen production, the electrolyser efficiency, the number of shutdowns, and the levelized cost of hydrogen. The results obtained are related to the case study and the chosen site, but some general considerations can be drawn.

\section{Method}

The system under study consists of a 1 MW PV power plant providing electrical energy to an alkaline electrolyser for hydrogen production.

Annual simulations of the hydrogen production were performed in Matlab for different electrolyser sizes expressed as a percentage of the PV nominal power and ranging from $20 \%$ to $100 \%$ with $5 \%$ steps.

5-minute power production data of one year of a solar PV plant (Fig. 1) taken from the NREL database [8] were scaled to the nominal power of the PV plant. Not all the PV energy can be used by the electrolyser due to the electrolyser technical limitations, in particular, its operating power range. Therefore, the energy that could not be used by the electrolyser was supposed to be released to the grid.

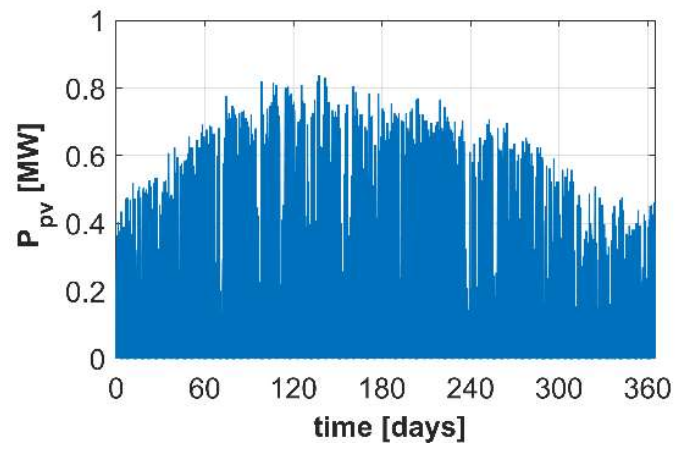

Fig. 1. PV power production.

An alkaline electrolyser model was adopted from [9] and scaled for the different sizes. An operating power range between $20 \%-100 \%$ of the nominal power was imposed as typical range for alkaline electrolyser. When PV power is below the lower power limit, the electrolyser is turned off while, over the maximum power limit, the excess of PV energy is released to the grid. The hydrogen production and the efficiency were calculated at each 5minute time step given the electrical power input and the nominal electrolyser power. Inside the model the cell current $\left(I_{\text {cell }}\right)$ and voltage $\left(V_{\text {cell }}\right)$ are determined by solving the system of Eqs. (1) and (2) by means of the Newton iteration method:

$$
\begin{gathered}
V_{\text {cell }}=V_{\text {rev }}+C_{1} \cdot \log _{10}\left(C_{2} \cdot I_{\text {cell }}+1\right)+R \cdot I_{\text {cell }} \\
\mathrm{P}=n_{\text {cell }} V_{\text {cell }} I_{\text {cell }}
\end{gathered}
$$

where $n_{\text {cell }}$ is the number of cells in series, and $\mathrm{P}$ is the power input to the electrolyser, that is the $\mathrm{PV}$ power minus the power required for the balance of the plant $\left(\mathrm{P}_{\mathrm{aux}}\right)$, expressed as a percentage of the total power, decreasing for increasing loads as in [10].
The coefficients of Eq. (1) are determined from the model adopted in [9] assuming an operating temperature of $70^{\circ} \mathrm{C}$ and a pressure of 5 bar. The resulting polarization curve is shown in Fig. 2.

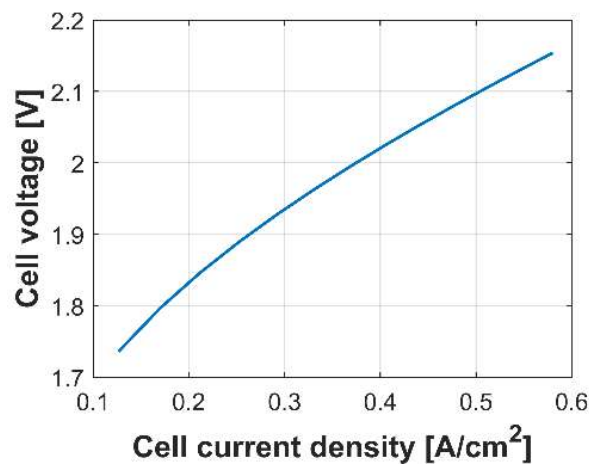

Fig. 2. Electrolyser's current - voltage curve.

The hydrogen production rate $\left(\dot{n}_{H_{2}}\right)$, expressed in $[\mathrm{mol} / \mathrm{s}]$, and the electrolyser efficiency $\left(\eta_{L H V}\right)$ are then calculated for each time step as:

$$
\begin{aligned}
& \dot{n}_{H_{2}}=\eta_{F} I_{\text {cell }} /(2 \mathrm{~F}) \\
& \eta_{L H V}=\dot{n}_{H 2} \mathrm{LHV} / \mathrm{P}
\end{aligned}
$$

where $\mathrm{F}$ is the Faraday constant, and LHV is the lower heating value of hydrogen $(241 \mathrm{~kJ} / \mathrm{mol})$. The Faraday efficiency, $\eta_{F}$, is expressed with a 5-parameters model, as a function of the current density ([9]).

The electrolyser efficiency, shown in Fig. 3 is a function of the load and has a maximum for a power input of about half the nominal power.

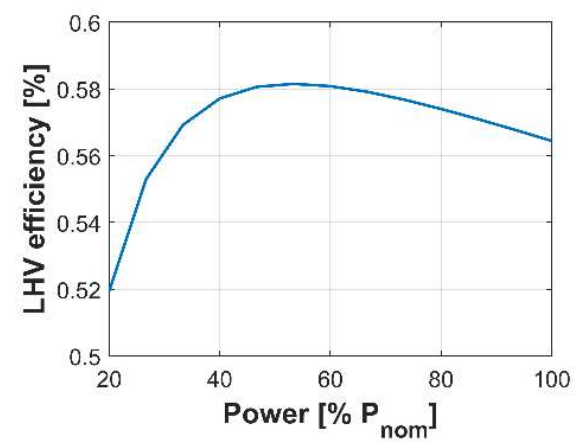

Fig. 3. Electrolyser efficiency as function of the power input.

For each simulation, the capacity factor, the number of shutdowns, the total operating time, and the percentage of PV energy used for water electrolysis are determined as important indicators of the electrolyser operation.

The levelized cost of hydrogen ( $\mathrm{LCOH}$ ) was evaluated for each electrolyser size as the sum of the contribution of the electrolyser cost (including annualized capital cost, operation and maintenance cost, and stack replacement cost), $\mathrm{LCOH}_{\text {electr }}$, and of the production cost of electrical energy, $\mathrm{LCOH}_{\mathrm{el}}$ en.

$\mathrm{LCOH}=\mathrm{LCOH}_{\text {electr }}+\mathrm{LCOH}_{\text {el en }}=\frac{C_{e l, a n n}}{V_{H 2, a n n}}+\frac{\mathrm{LCOE} \cdot E_{P V, a n n}}{V_{H 2, a n n}}$

where $\mathrm{E}_{\mathrm{PV} \text {,ann }}$ is the annual electrical energy produced by the $\mathrm{PV}$ plant and $\mathrm{V}_{\mathrm{H} 2 \text {,ann }}$ is the annual hydrogen 
production, expressed in [kg]. A levelized cost of energy (LCOE) of $60 € / \mathrm{MWh}[11]$ is considered for the PV energy production.

The annualized cost for the electrolyser was evaluated as:

$C_{e l, a n n}=\mathrm{CRF}\left[C_{c a p, e l}+C_{\text {rep }, e l}(1+\mathrm{IR})^{-10}\right]+C_{O \& M, e l}$

where $C_{\text {cap,el }}$ is the electrolyser capital cost, $C_{\text {rep,el }}$ is cost of stack replacement (supposed to be after 10 years), and $C_{O \& M, e l}$ is the operation and maintenance cost. The capital recovery factor, $\mathrm{CRF}$, is given by:

$$
\mathrm{CRF}=\mathrm{IR}(1+\mathrm{IR})^{\mathrm{Lt}} /\left[(1+\mathrm{IR})^{\mathrm{Lt}}-1\right]
$$

Table 1 shows the main assumptions for the economic evaluation.

Table 1. Economic parameters.

\begin{tabular}{|c|c|c|}
\hline Parameter & Value & Ref. \\
\hline Lifetime, Lt & 20 years & {$[5]$} \\
\hline Interest rate, IR & $5 \%$ & {$[5]$} \\
\hline Specific capital cost & $750 € / \mathrm{kW}$ & {$[12]$} \\
\hline \begin{tabular}{c} 
O\&M cost, $C_{O \& M, e l}$ \\
\hline $\begin{array}{c}\text { Stack replacement } \\
\text { cost (1 repl.) }\end{array}$
\end{tabular} & $3 \% \%$ year of the capital cost & {$[13]$} \\
\hline
\end{tabular}

LCOH defined by Eq. 5 assumes to have no gain from selling the PV energy not used by the electrolyser.

Then, in order to take into account the possible gain from selling the electrical energy surplus to the grid, a new $\mathrm{LCOH}\left(\mathrm{LCOH}^{*}\right)$ was evaluated assuming a selling price for the energy released to the grid, by Eq. 8:

$$
\mathrm{LCOH}^{*}=\mathrm{LCOH}-p_{\text {sell }} \cdot \frac{E_{P V, a n n}-E_{e l, a n n}}{V_{H 2, a n n}}
$$

Several selling prices $\left(p_{\text {sell }}\right)$ for the energy released to the grid were considered to see how they can affect the $\mathrm{LCOH}$ and the choice of the electrolyser size.

\section{Results and discussion}

Results show that the average operating power of the electrolyser (Fig. 4) initially increases with the nominal power since the maximum power limit increases allowing the electrolyser to operate at higher powers. At higher nominal powers, the increase of the average operating power becomes less marked. Indeed, most of the highest PV power values can be already completely absorbed by electrolysers with a lower nominal power; thus, the slight increase of the average power is mainly due to the cut of the lowest PV powers.

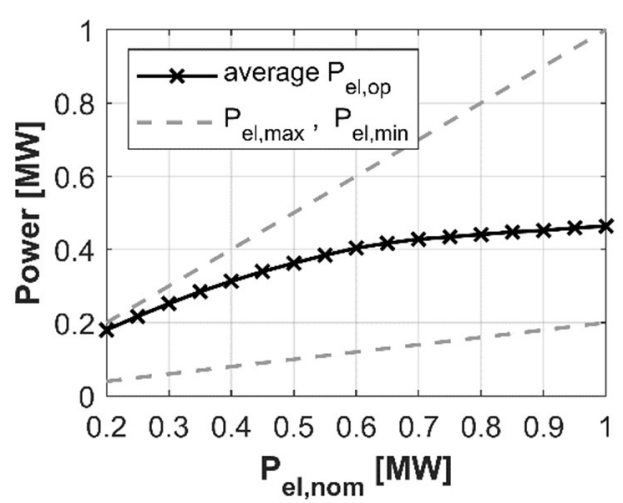

Fig. 4. Average operating power of the electrolyser and operating power limits as function of the electrolyser nominal power.

Furthermore, at lower nominal powers, the electrolyser operates averagely nearer to its maximum power at lower efficiencies (Fig. 5). At higher nominal powers, the electrolyser works more often at part-load conditions and averagely at higher efficiencies.

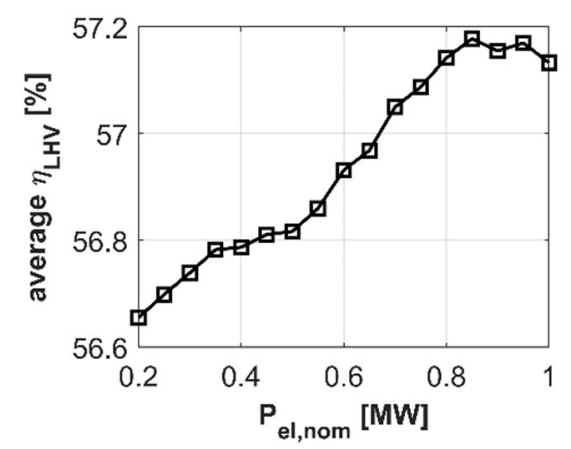

Fig. 5. Average efficiency of the electrolyser.

On one hand, electrolysers with lower nominal powers operate for a longer time and are closer to the nominal power, thus showing higher capacity factors as shown in Fig. 6. On the other hand, they can use only a small part $\left(E_{e l}\right)$ of the total energy produced by the PV plant $\left(E_{p v}\right)$.

The maximum percentage of energy used by the electrolyser is reached for a nominal power of $0.7 \mathrm{MW}$. At higher power values, the cut of the lowest PV powers due to the greater minimum power limit of the electrolyser is not compensated by the increase of the maximum power limit.

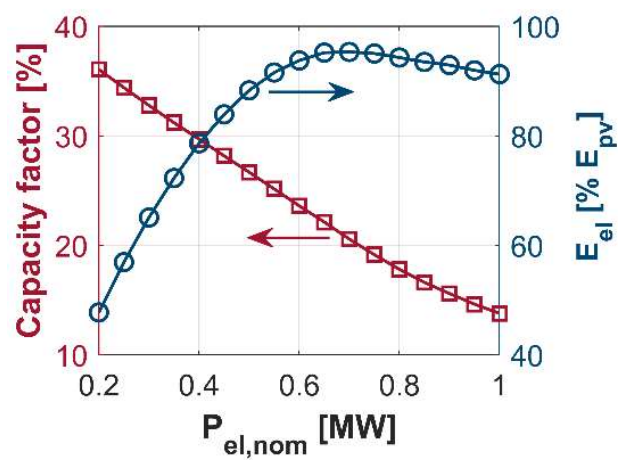

Fig. 6. Capacity factor and percentage of PV energy used by the electrolyser. 
The annual hydrogen production (Fig. 7) increases with the nominal power of the electrolyser until reaching a maximum for a nominal power of $0.7 \mathrm{MW}$, the same value that maximizes the amount of PV energy used by the electrolyser. However, at this nominal power, the electrolyser has a low capacity factor (Fig. 6), slightly higher than $20 \%$ that can increase the payback time.

Nominal power higher than $0.7 \mathrm{MW}$ showed even lower operating time. These electrolyser sizes should be therefore avoided from all the points of view.

The results obtained suggest that the best choice for the electrolyser size is a trade-off between the maximization of the hydrogen production and the maximization of the electrolyser capacity factor.

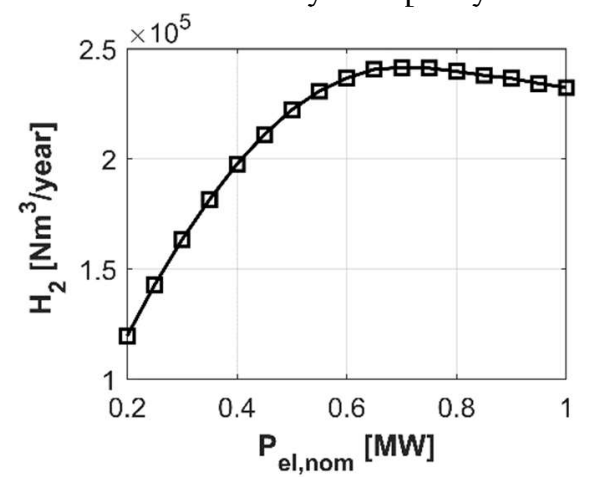

Fig. 7. Annual hydrogen production.

Another important parameter is the number of electrolyser on/off, which must be as low as possible to avoid fast performance degradation and consequent reduction of the stack lifetime.

Due to the nature of solar energy, the electrolyser is shut down at least one time per day. However, the number of on/off can be higher, especially in the case of cloudy days. Results show an increase in the annual number of on/off with the nominal power of the electrolyser (Fig. 8). Indeed, the PV power becomes more frequently lower than the minimum operating power in the case of higher nominal powers.

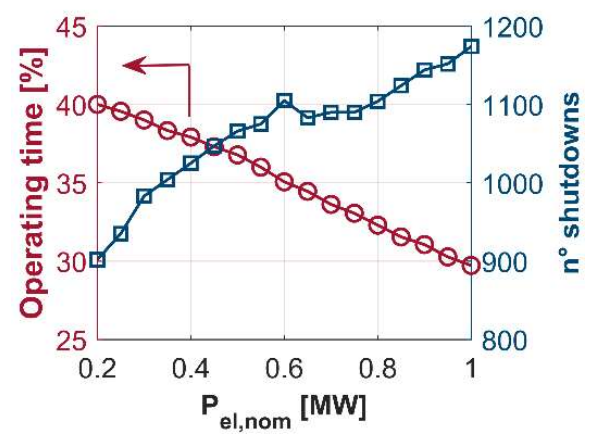

Fig. 8. Operating time percentage and annual number of shutdowns.

The levelized cost of hydrogen (Fig. 9) reaches a minimum at a nominal power of the electrolyser of 0.55 $\mathrm{MW}$ for a LCOE of $60 € / \mathrm{MWh}$. Indeed, on the one side, the contribution associated with the electrolyser cost grows with the size since the increasing annualized cost is not balanced by the increase in hydrogen production (because the capacity factor of the electrolyser decreases).
On the other side, the contribution of the electrical energy cost to $\mathrm{LCOH}$ decreases with the electrolyser nominal power since the cost of the electrical energy produced by the PV plant is constant while the annual hydrogen production increases for nominal powers lower than 0.7 MW.

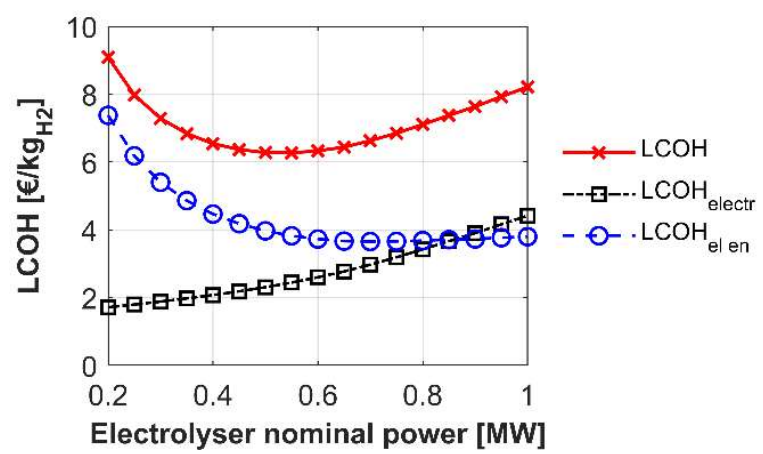

Fig. 9. Levelized cost of hydrogen (LCOH) and electrolyser- and electrical energy contributions ( $\mathrm{LCOE}=60 € / \mathrm{MWh})$.

The electrolyser nominal power giving the minimum LCOH increases with LCOE (Fig. 10). The reason is that the weight of the contribution of the production cost of the electrical energy to LCOH increases with LCOE and has decreasing trend with the electrolyser nominal power.

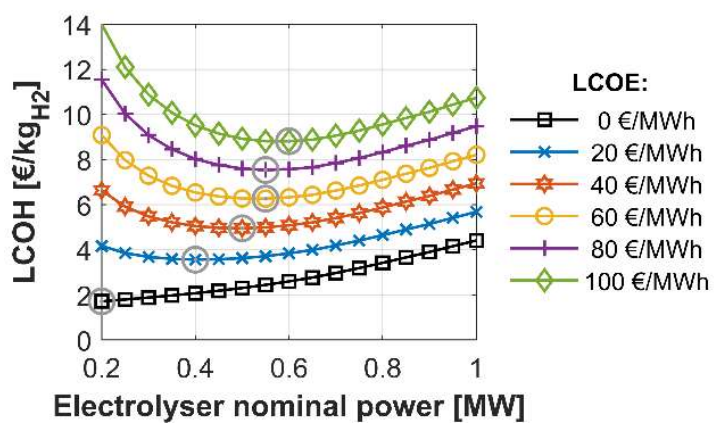

Fig. 10. Levelized cost of hydrogen $(\mathrm{LCOH})$ for different LCOEs.

Finally, $\mathrm{LCOH}^{*}$ (Fig. 11) shows a minimum for intermediate values of the nominal power of the electrolyser when the selling price of electrical energy is lower than LCOE. As the nominal power of the electrolyser increases, the beneficial effect of the selling price to reduce the $\mathrm{LCOH}$ decreases because the amount of electrical energy sold decreases.

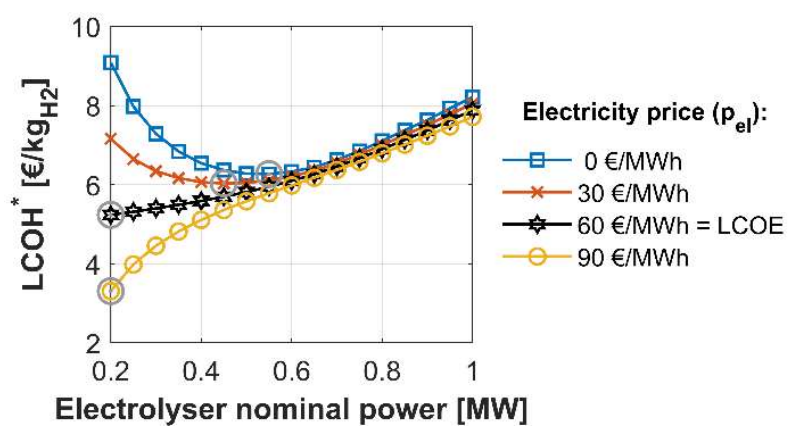

Fig. 11. Levelized cost of hydrogen $\left(\mathrm{LCOH}^{*}\right)$ taking into account different selling price of electrical energy ( $\mathrm{LCOE}=60 € / \mathrm{MWh})$. 


\section{Conclusion}

The hydrogen production by an alkaline electrolyser fed by a 1 MW PV plant was simulated for different electrolyser nominal powers. For the studied case, the annual hydrogen production was maximized for a nominal power of the electrolyser of $0.7 \mathrm{MW}$. However, the increase of the electrolyser size leads to a decrease in the electrolyser capacity factor. Furthermore, the number of electrolyser shutdowns increases at higher electrolyser nominal powers leading to faster degradation. From an economic point of view, higher electrolyser sizes are related to higher capital costs that are not compensated by the increase in hydrogen production.

Assuming to not have a gain from selling the PV energy surplus, the minimum $\mathrm{LCOH}$ was obtained for an intermediate electrolyser nominal power, lower than that maximizing the hydrogen production. Even if the results are obtained for a specific site, some outcomes can be generalized. The best choice for the electrolyser size should be the result of a trade-off between the maximization of hydrogen production and the need of limiting the number of shutdowns and of having a sufficiently high capacity factor of the electrolyser in order to keep the LCOH down.

\section{References}

1. E. Bargiacchi, M. Antonelli, U. Desideri, Energy 183, 1253-1265 (2019).

2. Á. Hernández-Gómez, V. Ramirez, D. Guilbert, Int. J. Hydrogen Energy 45, 14625-14639 (2020).

3. A. Ursúa, E.L. Barrios, J. Pascual, I. San Martín, P. Sanchis, Int. J. Hydrogen Energy 41, 12852-12861 (2016).
4. F. Gutiérrez-Martín, L. Amodio, M. Pagano, Int. J. Hydrogen Energy 46, 29038-29048 (2021).

5. C. Schnuelle, T. Wassermann, D. Fuhrlaender, E. Zondervan, Int. J. Hydrogen Energy 45, 29938-29952 (2020)

6. J. Yates, R. Daiyan, R. Patterson, R. Egan, R. Amal, A. Ho-Baille, N.L. Chang, Cell Reports Physical Science 1, 100209 (2020).

7. A. Khalilnejad, A. Abbaspour, A.I. Sarwat, Int. J. Hydrogen Energy 41, 11884-11894 (2016).

8. NREL, Solar Power Data for Integration Studies. https://www.nrel.gov/grid/solar-powerdata.html (accessed 29 March 2021).

9. M. Sánchez, E. Amores, L. Rodríguez, C. ClementeJul, Int J Hydrogen Energy 43, 20332-20345 (2018).

10. A. Liponi, G.F. Frate, A. Baccioli, L. Ferrari, U. Desideri, Green hydrogen from wind energy: mitigation of operating point fluctuations, in: Proceedings of ECOS - The 34th International Conference, Italy (2021).

11. IRENA, Renewable Power Generation Costs in 2019 , Abu Dhabi, 2020.

12. J. Proost, Int J Hydrogen Energy 44, 4406-4413 (2019).

13. J. Armijo, C. Philibert, Int J Hydrogen Energy 45, 1541-1558 (2020).

14. B. Olateju, A. Kumar, M. Secanell, Int J Hydrogen Energy 41, 8755-8776 (2016). 